\title{
Differential detection of alternatively spliced variants of Ciz1 in normal and cancer cells using a custom exon-junction microarray
}

\author{
Faisal A Rahman ${ }^{1,2}$, Naveed Aziz ${ }^{1}$, Dawn Coverley ${ }^{1 *}$
}

\begin{abstract}
Background: Ciz1 promotes initiation of mammalian DNA replication and is present within nuclear matrix associated DNA replication factories. Depletion of Ciz1 from normal and cancer cells restrains entry to $S$ phase and inhibits cell proliferation. Several alternative splicing events with putative functional consequences have been identified and reported, but many more variants are predicted to exist based on publicly available mRNAs and expressed sequence tags.

Methods: Here we report the development and validation of a custom exon and exon-junction microarray focused on the human CIZ1 gene, capable of reproducible detection of differential splice-variant expression.

Results: Using a pair of paediatric cancer cell lines and a pool of eight normal lines as reference, the array identified expected and novel CIZ1 splicing events. One novel variant (delta 8-12) that encodes a predicted protein lacking key functional sites, was validated by quantitative RT-PCR and found to be over-represented in a range of other cancer cell lines, and over half of a panel of primary lung tumours.

Conclusions: Expression of CIZ1 delta 8-12 appears to be restricted to cancer cells, and may therefore be a useful novel biomarker
\end{abstract}

\section{Background}

Ciz1 (Cip1 interacting zinc-finger protein 1) is a nuclear protein that appears to be encoded by 17 exons that map to chromosome 9q34 in man (Unigene Hs.212395). Ciz1 is expressed in a wide range of tissues [1] and contains $\mathrm{N}$-terminal polyglutamine repeats, 3 zinc finger motifs, one matrin 3 domain at the C-terminal end, 2 putative nuclear localization signals and a number of cyclin-dependent kinase phosphorylation sites and cyclin-binding motifs [2]. Ciz1 was first identified through its interaction with p21/Cip1 [3], a cyclindependent kinase (CDK) inhibitor involved in regulation of the cell cycle and in cellular differentiation. We identified Ciz1 in a cell-free system that reconstitutes initiation of DNA replication, under the regulation of cyclin A/CDK2 [4]. Using cell-free and cell-based approaches we showed that Ciz1 stimulates initiation of DNA

\footnotetext{
* Correspondence: dc17@york.ac.uk

'Department of Biology, University of York, UK

Full list of author information is available at the end of the article
}

replication in late G1 phase nuclei [5] and that it cooperate with cyclin $\mathrm{E}$ and $\mathrm{A}$ in order to execute its function in DNA replication [2]. Further detailed analysis revealed that DNA replication activity resides in the $\mathrm{N}$-terminal half of Ciz1, while the protein becomes tethered to non-chromatin nuclear matrix structures through $\mathrm{C}$-terminal sequences that play a role in its localization to DNA replication factories [6]. Taken together the data suggest that Ciz1 plays a linker role between the DNA replication machinery and the subnuclear structures that organize their function.

CIZ1 is a hormone-responsive gene $[7,8]$ and is thought to play a role in the development or progression of oestrogen responsive tumours. It has also been linked with certain paediatric cancers $[1,9]$, where alternatively spliced transcripts are prevalent. Similarly, inappropriate expression of alternatively spliced transcript has been reported to occur in brain tissue from Alzheimer patients [10]. Previously, we surveyed over $600 \mathrm{CIZ1}$ expressed sequence tags that map to the CIZ1 Unigene cluster [9], to overview alternative splicing during development and disease. This 
highlighted three exons in the $5^{\prime}$ end of Ciz1 that are commonly alternatively spliced and potentially mis-spliced under some circumstances, and suggested a range of other rarer splicing events.

In this study our aim is to look at CIZ1 variant expression in order to validate previously reported alternative splicing (AS) events, and to discover new ones. We chose to use an exon junction array because commonly used methods for splicing analysis such as RT-PCR, cDNA primer extension and nuclease protection mapping are extremely labour intensive for highly spliced genes, particularly when analysis of a large number of samples is expected. Therefore, we have developed an exon junction-array focused on the human CIZ1 gene that is capable of detecting all known variants and hypothetical variants of CIZ1. We present results generated with this exon array-tool, initially using a pair of paediatric cancer cell lines. This identified several novel AS events including one cancerrestricted novel variant that we chose to validate further. The functional implications of expression of this variant (which has partial deletion of exon 8 and exon 12 and skipping of exon 9, 10 and 11) are discussed and potential applications highlighted.

\section{Methods}

\section{Array and probe design}

We designed oligonucleotide probes with extensive coverage for CIZ1 mRNAs and predicted variants. These include probes homologous to exons, observed junctions, hypothetical junctions and retained intron sequences (Fig. 1). The array also includes probes homologous to other genes that either emerged from our biochemical studies as Ciz1 interacting partners, have roles in cell cycle predicted to interact with Ciz1 or are known to be involved in pathology of sporadic cancers (additional file 1 Table S1). For $5^{\prime}$ untranslated sequences we included probes that cover possible alternative first exons. These are defined as exon 1a (in NCBI Reference Sequence: NM_012127.2 and NM_001131015.1) and exon 1c (NM_001131017.1, NM_001131018.1 and NM_001131016.1). Other alternatively used exon $1 \mathrm{~s}$ were predicted by AceView (additional file 2 Figure S1) and confirmed by us when searching CIZ1 EST transcript against the BLAT CIZ1 assembly [11]http://genome.ucsc.edu/, and designated exon $1 b$ and $1 d$ (additional file 3 Figure S2). To our knowledge inclusion of exon $1 b$ and $1 d$ has not been verified by inclusion in a full-length human CIZ1 transcript.

To allow for a reasonable trade off between signal intensity and specificity, we restricted length of the probes to 40 nucleotides [12]. To increase specificity of probes, interspersed repeats and low complexity DNA sequences were masked (repeats have been replaced by Ns) using repeat masker [13] http://www.repeatmasker. org. The masked sequences were then searched for similarity against BLAT and areas which showed significant

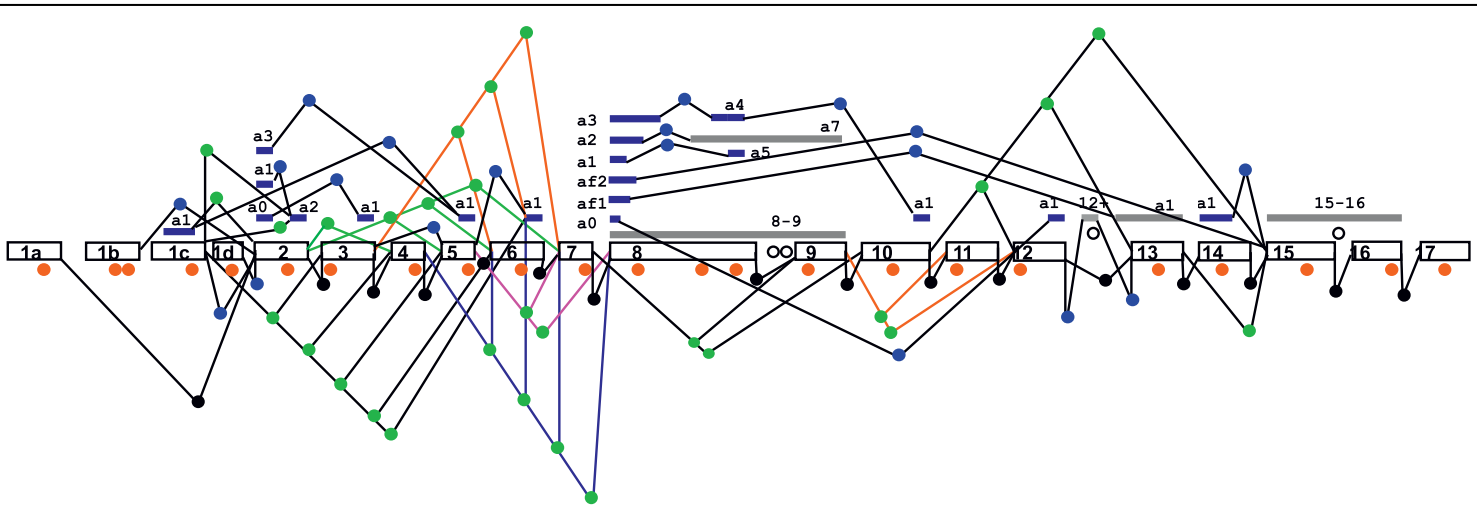

\begin{tabular}{|ll|}
\hline Most common predicted exons & - Exon -junction probes \\
Less common alternative exons & - Rare exon junction probes (observed) \\
Rare alternative exons containing intronic sequences & - Exon-junction probes (hypothetical) \\
Exon probes & O Retained intron probes \\
& \\
\hline
\end{tabular}

Figure 1 Schematic representation of the location of human $\mathrm{CIZ1}$ probes, designed to detect expression of the most common predicted exons, less common alternative exons, rare alternative exons containing intronic sequences, most common and observed rare exon-junctions and a selection of hypothetical junctions. For junction probes between most common predicted exon, - indicates the junction such as Ciz1ex2-ex3. For junction probes between less common predicted exons, such as Ciz1 exon 2 alternative 1 and exon 2 alternative 2, the probe designation will be defined as Ciz1-ex2a1-ex2a2. Probe sequences are given in additional file 1 Table S1. 
similarity were excluded. For junction probes, we employed the sliding window method where the $40 \mathrm{nt}$ can slide across the target junction to allow for near similarity in melting temperatures [12]. Probes were then searched for similarity using BLAST non-redundant database with expectation value of $10 \mathrm{http}$ ://blast. ncbi.nlm.nih.gov/Blast.cgi[14]. Probes with more than 20 contiguous identical bases to non-self transcript were excluded. For all probes, exclusion criteria were set to include those with hairpin structures with stem exceeding 9 bases as well as those with self complementarities of more than 6 bases [15]. However, due to the constraints of the exon junctions, these parameters could not be applied to all probes. As the CIZ1 gene is rich in GCs, the target $\mathrm{T}_{\mathrm{m}}$ was set not to exceed $85^{\circ} \mathrm{C}$ (additional file 1, Table S1). Probes were synthesized by Operon Biotechnologies $\mathrm{GmbH}$, Cologne, Germany. The oligonucleotide probes were printed on Nexterion Slides E (Schott) using a QArrayMini (Genetix) as follows: 5 replicates were printed for each probe in a random pattern including buffer as negative controls. Two slides from each batch of printing were analysed for spot quality using Ribogreen (Molecular Probes). Prior to hybridisation, each slide was washed at room temperature as follows: $1 \times 5 \mathrm{~min}$ in $0.1 \%$ Triton $\mathrm{X}-100,2 \times 2 \mathrm{~min}$ in $1 \mathrm{mM} \mathrm{HCL}, 1 \times 10 \mathrm{~min}$ in $100 \mathrm{mM}$ KCL. Slides were subsequently blocked by incubation in $1 \times$ Nexterion $^{\circ}$ Blocking E (Schott) for $15 \mathrm{~min}$ and then dried by centrifugation. Microarray data associated with this paper was submitted to ArrayExpress (accession: E-MEXP-2331) with the experiment name 'Differential detection of alternatively spliced variants of Ciz1'.

\section{Cell culture and RNA extraction}

A pool of eight stable cell lines of non-tumour origin were used as a reference control, (IMR90, WI38, HEK293, MRC5, HFL1, ASF-4-1, TIG-2M-30 and RPM1788, Table 1). Cell lines were obtained from the

Table 1 Cell lines used in this analysis

\begin{tabular}{lll}
\hline Cell line & Derivation & Reference \\
\hline TTC466 & Ewings Tumour & {$[23]$} \\
\hline SKNMC & PNET & {$[24]$} \\
\hline H727 & Human bronchial carcinoid & {$[25]$} \\
\hline IMR90 & Human embryonic lung & {$[26]$} \\
\hline WI38 & Human embryonic lung & {$[27]$} \\
\hline HEK293 & Human embryonic kidney & {$[28]$} \\
\hline MRC5 & Human embryonic lung & {$[29]$} \\
\hline HFL1 & Human embryonic lung & {$[30]$} \\
\hline ASF-4-1 & Skin fibroblast from upper arm & {$[31]$} \\
\hline RPMI 1788 & normal blood, lgM secreting & {$[32]$} \\
\hline TIG-2M-30 & muscle, normal diploid fibroblast & {$[33]$} \\
\hline
\end{tabular}

European Collection of Cell Cultures (ECACC) and the Japanese Collection of Research Bioresources (JCRB). Cells were cultured exactly as recommended. Total RNA was extracted using TRIZOL as recommended by the manufacturer (Invitrogen). RNA concentration was quantified using nanodrop spectrophotometer and equal concentrations from each of the eight samples were pooled together. SKNMC and TC466 Ewing tumour cell lines were used as test samples to validate the array and were handled as described previously [9].

\section{Preparation and labeling of templates}

$20 \mu \mathrm{g}$ of pooled RNA reference control or test samples (SKNMC and TTC466) were reversed transcribed as described and labeled using Invitrogen protocol (SuperScript ${ }^{\mathrm{Tm}}$ Plus Indirect cDNA labeling System). Labelled reference and test sample $(20 \mu \mathrm{l}$ each) were combined in an amber tube, mixed and heated at $99^{\circ} \mathrm{C}$ for three minutes before hybridization.

\section{Prehybridization and Hybridization}

Prior to hybridization slides were prepared as mentioned previously. Hybridization was carried out in a final volume of $40 \mu \mathrm{l}$ in Corning hybridization chambers, in a water bath at $42^{\circ} \mathrm{C}$ for $16-24 \mathrm{~h}$. Microarray slides were then washed twice in $1 \times$ SSC with $0.1 \%$ SDS for five minutes, once in $0.1 \times$ SSC with $0.1 \%$ SDS for 5 minutes and a final wash in $0.05 \times$ SSC for five minutes. Slides were transferred to a centrifuge tray and spun for three minutes at $2000 \mathrm{rpm}$ at room temperature to dry.

\section{Image acquisition and data analysis}

Slides were scanned using GenePix 4000B microarray scanner and images were analyzed using GenePix software where a grid layout was applied to the features. Data analysis was performed using ArrayAssist software where background correction was done using foreground-background (FG-BG). Features with signal intensities less than 2.5 fold of the mean background signal were removed, and ratios were normalized using housekeeping gene normalization [16] and were averaged and log transformed.

\section{Reverse-Transcriptase PCR}

cDNA was generated using superscript III Synthesis Kit according to manufacturer's instructions (Invitrogen). Normalization of input cDNA was done using Actin forward (5'CAACCGCGAGAAGATGACC3') and reverse primers (5'TCCAGGGCGACGTAGCA CA3') as endogenous control. For CIZ1 sequences spanning exon 8 to exon 13, primers Ciz1-ex8F (5'CTCCAGGGCAGTTACAGGAC3') and Ciz1-ex13R2 (5'TGCGAGGGGTT TTGAAGTAG3') were PCR amplified at $58^{\circ} \mathrm{C}$ annealing temperature using phusion high-fidelity DNA polymerase 
(Finnzymes). For the variant with partial deletion of exon 8 and exon 12 and skipping of exon 9, 10 and 11, splice junction specific primers were used. Forward primer, Ciz1jex8-ex12F (5'CTCCAGGGCAGTTACAGGAC3') and reverse primer hCiz1-Jex13-ex14R (5'CTCAAGCGACTTCAGCTCCT3') were PCR amplified at $60^{\circ} \mathrm{C}$ annealing temperature using Taq polymerase (New England Biolab (NEB). For alternative exon $1 \mathrm{~s}$, forward primers Ciz1ex1b (5'CAGACGGACCTTGGT CTCC3'), Ciz1-exon 1c (5'GCGACTTGAGCGTTGAGG3') and Ciz1-ex1d (5' GGCGG TGGTGGAGAGAAG3') were all combined with reverse primer Ciz1-ex5R (5'CGATTGG GGGTGGTAGAGG3') in separate reactions. Taq polymerase (NEB) was used for amplification at annealing temperatures of $60^{\circ} \mathrm{C}, 62^{\circ} \mathrm{C}$ and $56^{\circ} \mathrm{C}$ respectively.

\section{Quantitative Real-Time PCR}

Reactions were carried out using an $\mathrm{ABI} 7000$, initial incubation at $95^{\circ} \mathrm{C} / 10 \mathrm{~min}$, followed by $95^{\circ} \mathrm{C} / 15 \mathrm{sec}$ and $1 \mathrm{~min}$ at $60^{\circ} \mathrm{C}$ for 40 cycles. Triplicate reactions were carried out in 96-well plates in a $25 \mu \mathrm{l}$ reaction volume containing $12.5 \mu \mathrm{l}$ cyber green Master Mix (Applied Biosystems), 20 ng cDNA and $200 \mathrm{nM}$ each of hCiz1Jex8-ex12F forward and hCiz1-Jex13-ex14R reverse. Primers used for normalization include, Actin F and Actin $\mathrm{R}$ as well as GAPDH forward (5'ATCCCATCACCATCTTCCAGG3') and reverse (5'GCATC GCC CCACTTGATTTTG 3').

\section{Results and discussion}

CIZ1 is represented by 865 GenBank entries that map to Unigene Build number 224, cluster Hs.212395. These mRNAs and ESTs were aligned cooperatively by AceView [17] to exclude redundancies producing 27 mRNA assemblies (additional file 2 Figure S1 1). These were then aligned to the gene sequence and exon boundaries and splice junctions identified. CIZ1 probes were designed from constitutive regions, alternative regions and observed exon-exon splice junctions. To allow for discovery of novel alternative splicing events, we also designed probes representing hypothetical exon-exon junctions. The array is designed to survey overall CIZ1 alternative splicing in different tissues, diseases and developmental stages, with the aim of substantiating observed alternative splicing features and identifying novel alternative splicing events. Here we discuss initial array results on the SKNMC PNET and TTC466 Ewing tumour cell lines.

Analysis of the technical replicates in this study showed that out of 26 exon probes the data was consistent and informative for 24 probes (92\%) for the SKNMC cell line and 23 probes $(88.5 \%)$ for TTC466 cell line. Data was not obtained for the CIZ1 exon 17 probe in either cell line suggesting a problem with the probe. For the remaining 3 (Fig. 2A, B), their failure was cell-line specific, possibly reflecting severe under or over expression outside the detection parameters of this analysis. For the $73 \mathrm{CIZ1}$ junction probes used here reproducible data was not available for 5 junctions observed in the EST databases and 14 that were rare or hypothetical junctions for SKNMC cells (Fig. 3). Similarly for TTC466, of the 73 junction probes, data was not available for 4 observed junctions and 17 rare or hypothetical junctions (Fig. 4). Three of the observed and 11 of the rare or hypothetical junctions were common to both cell lines. We consider these figures to be within the expected range due to the constraints inherent in designing probes for exon junctions, coupled with the fact that some of the hypothetical junctions may never exist in reality. Of greater interest is the hypothetical junctions that were consistently represented in the transcriptome of these two cell lines. These are ex1ca1-ex2, ex1c-ex2a2, ex1c-ex4, ex1c-ex6, ex2-ex7 and ex7-ex10 (depicted graphically in additional file 3 Figure S2), indicating an unanticipated level of AS around untranslated exons 1 and 2 .

Analysis of 3 technical replicates for Ewings tumour cell line SKNMC showed that CIZ1 exon $1 b$ is up-regulated and CIZ1 intron 12 (alternative exon) is down regulated relative to the reference template, although both fall only just within inclusion criteria of 1.5 fold change. When compared to 2 further biological replicates (isolated from separate population of SKNMC cells), neither probe proved to be consistently different to the reference template (Table 2 and Fig. 2A). However similar analysis of cell line TTC466 also indicated differential expression of the alternative exon $1 \mathrm{~s}$. In fact CIZ1 exon 1c was considerably up-regulated in all three biological replicates (Table 3), with an SEM of 0.355135 between the three technical replicates (Fig. 2B). Moreover among the exon 1c-junction probes, 4 were consistently up-regulated relative to the reference control in all 3 biological replicates and 3 of these were among the top 4 ranked products [18] (additional file 4 Table S2). This analysis revealed splicing of exon $1 c$ onto exon 2 (variant 2), exon 4 and exon 6 and splicing of alternative exon 1c (1ca1) onto exon 2 (Table 3 and Fig. 3).

Other genes in this study that were overrepresented in all technical and biological replicates were RB1 and VPS72 for SKNMCS cell line, and CDC2, CDC6, TNRC9 and DDX17 in TTC466 cells (additional files 6 and 7 Tables S4-S13). VPS72 is an interacting partner of EWSR1 gene [19]. RB1, CDC2 and CDC6 may share the same pathways as CIZ1 gene. Expression of these genes needs to be validated and their function with regard to Ciz1 further analysed. 


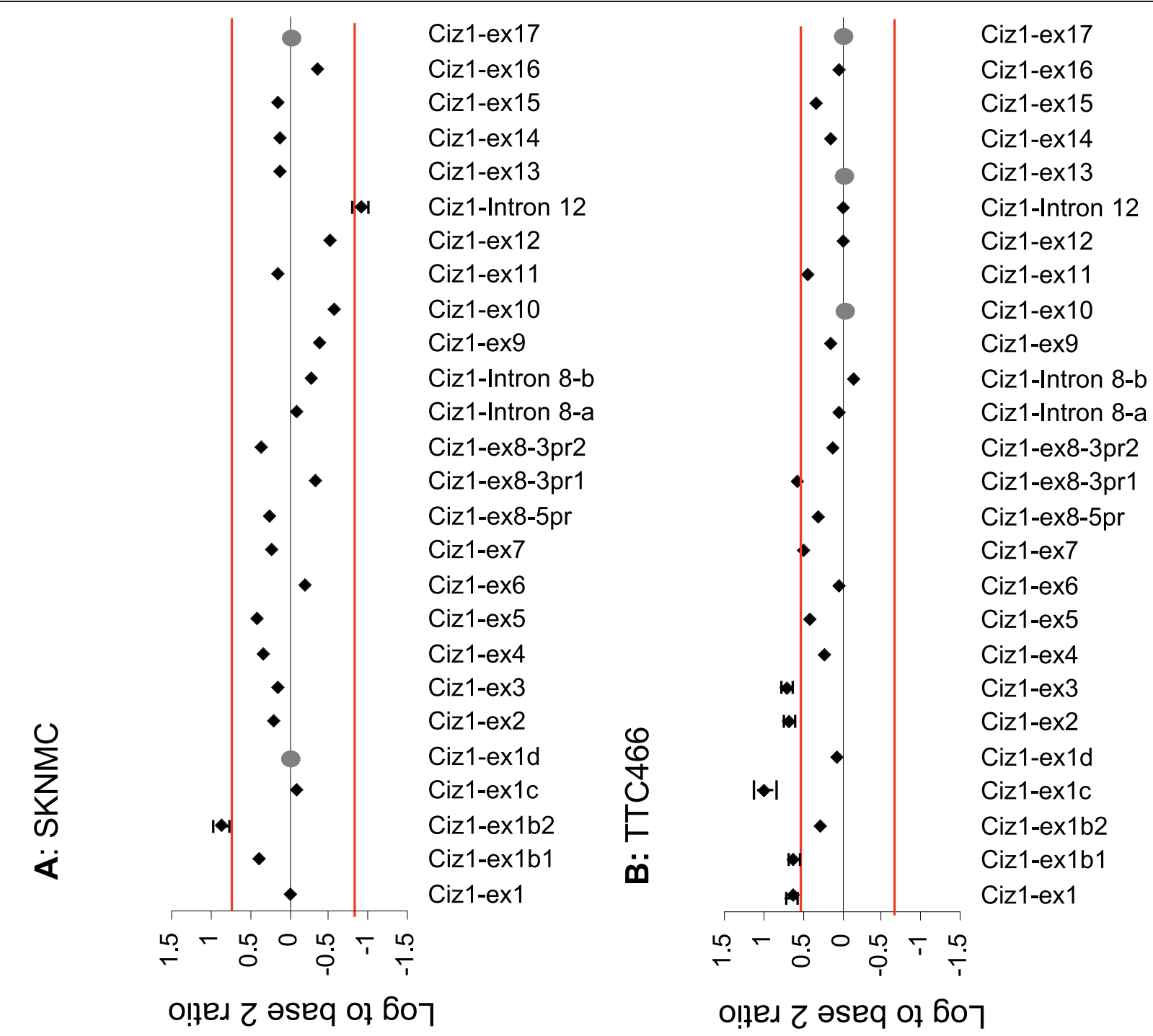

Figure 2 Expression of $\mathrm{CIZ} 1$ in paediatric cancer cell lines revealed by hybridization to $\mathrm{CIZ} 1$ exon probes. A: $\mathrm{CIZ1}$ exon expression in primitive neural ectodermal tumour (PNET) cell line SKNMC. B: CIZ1 exon expression in Ewing tumour TTC466 cell line. Plots show the average of three technical replicates expressed as $\log ^{2}$ of the ratio between signal generated by the test sample and signal generated by reference RNA. Change is considered to be significant where this fall outside 0.58 or -0.58 and therefore exceed 1.5 fold relative to control. Bars indicating SEM are shown for probes included by these selection criteria. No reliable data was achieved for probes indicated with a grey dot.

\section{Expression of alternative exon $1 \mathrm{~s}$}

We surveyed CIZ1 ESTs using BLAT [11] to review alternative usage of exon 1. This showed that CIZ1 exon $1 c$ is supported by $4 \mathrm{mRNAs}$ and 139 ESTs which exceeds by far the number of mRNAs and ESTs supporting other exon $1 \mathrm{~s}$. The second most common is exon $1 b$ supported by 16 ESTs. This analysis might not reflect the true nature of exon 1 usage due to possible bias towards 3 ' exons. In this study, we detected over-expression of the novel alternative exon $1 b$ in technical replicates of both SKNMC and TTC466 cell lines (Tables 2 and 3), however no change was observed in biological replicates of the two cell lines. On the other hand for exon 1c, array results showed consistent up-regulation in TTC466 cell line as well as the junctions of exon $1 c$ and other exons (2, 4 and 6). Taken together these results suggest that exon $1 c$ is the most widely used alternative exon 1 for the CIZ1 gene. Protein translation for alternative usage of exon $1 \mathrm{~s}$ on the background of full length Ciz1 showed that exon $1 c$ and $1 d$ would encode the same protein. On the other hand translation for exon $1 b$ showed there are two additional predicted methionines upstream of the usual predicted methionine in exon 2, adding an extra 28 amino-acids to the N-terminal end of the Ciz Protein. 5' UTR selection has been shown to affect alternative splicing of downstream exons. For example, for oestrogen receptor $\beta$ it has 


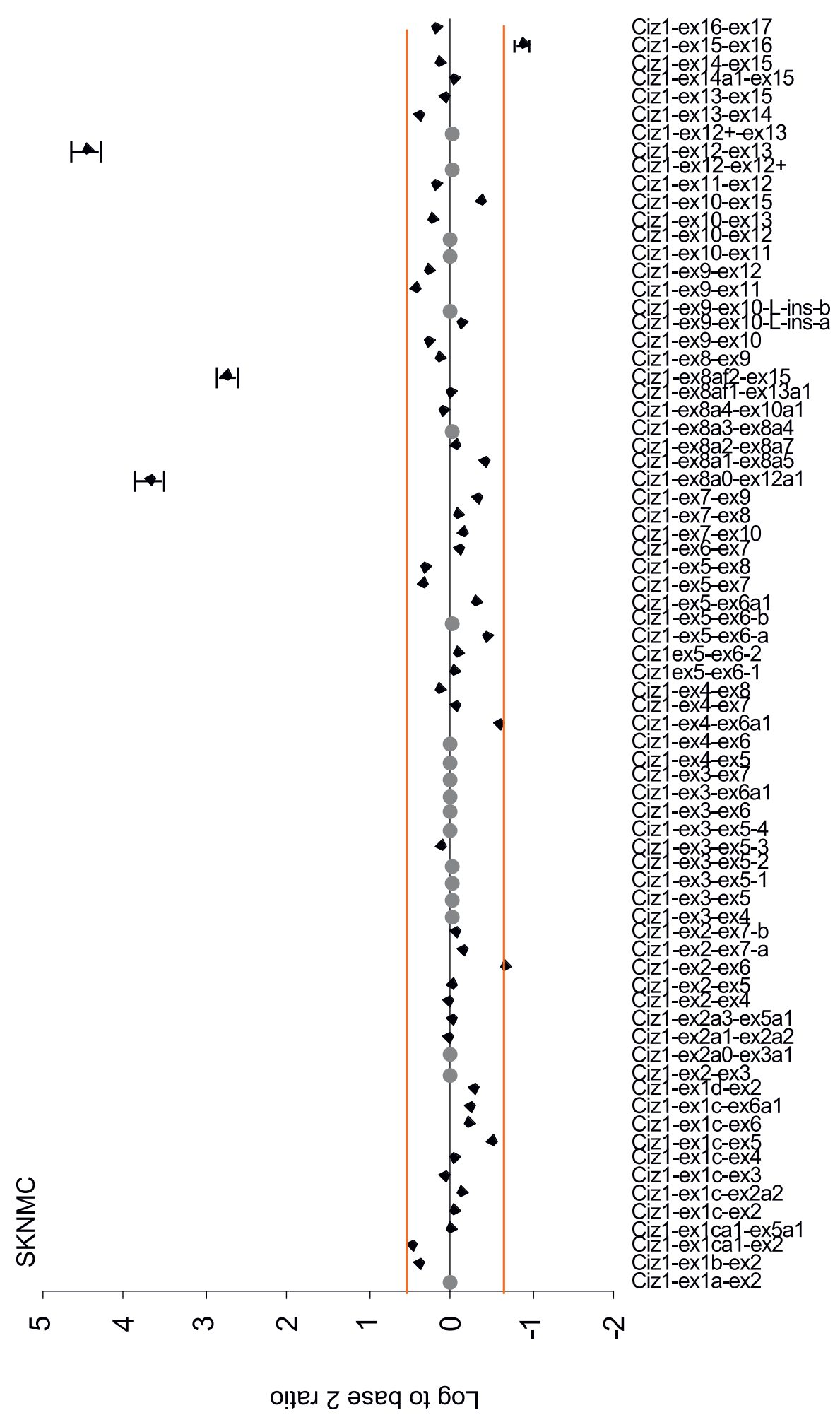

Figure 3 Expression of $\mathrm{CIZ1}$ in paediatric cancer cell lines revealed by hybridization to $\mathrm{CIZ1}$ exon-junction probes for SKNMC PNET cell line. Plots show the average of three technical replicates expressed as $\log ^{2}$ of the ratio between signal generated by the test sample and signal generated by reference RNA. Change is considered to be significant where this fall outside 0.58 or -0.58 and therefore exceed 1.5 fold relative to control. Bars indicating SEM are shown for probes included by these selection criteria. No reliable data was achieved for probes indicated with a grey dot. 


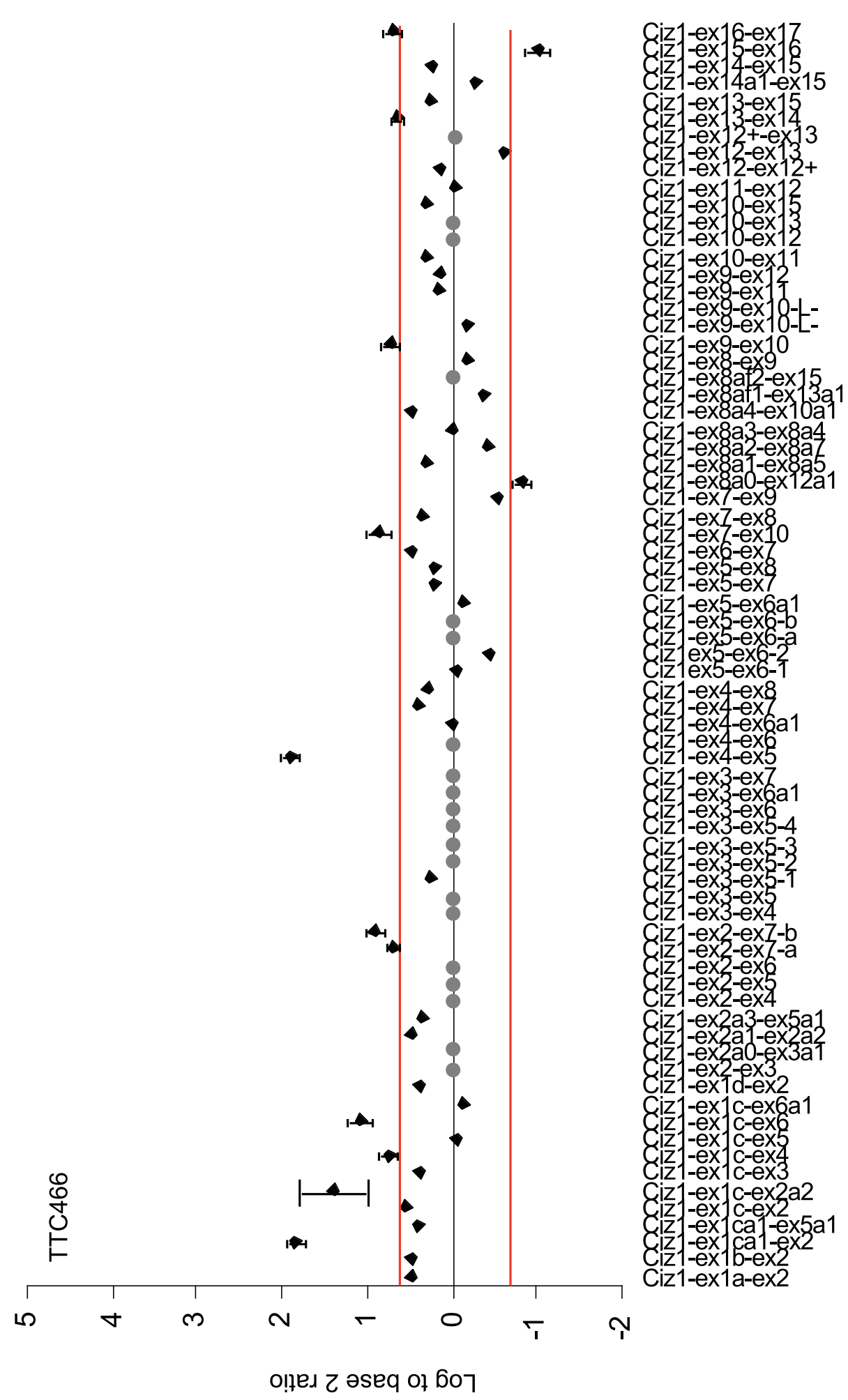

Figure 4 Expression of $\mathrm{CIZ1}$ in paediatric cancer cell lines revealed by hybridization to $\mathrm{CIZ1}$ exon-junction probes for TTC466 Ewing tumour. Plots show the average of three technical replicates expressed as $\log ^{2}$ of the ratio between signal generated by the test sample and signal generated by reference RNA. Change is considered to be significant where this fall outside 0.58 or -0.58 and therefore exceed 1.5 fold relative to control. Bars indicating SEM are shown for probes included by these selection criteria. No reliable data was achieved for probes indicated with a grey dot. 
Table 2 Summary of $\mathrm{CIZ1}$ alternative splicing in SKNMC cell line

\begin{tabular}{llll}
\hline SKNMC exon probes & Average of 3 technical replicates (B1) & Biological replicate 2 & Biological replicate $\mathbf{3}$ \\
\hline Ciz1-ex1b2 & + & $=$ & $=$ \\
\hline Ciz1-intron 12 & - & $=$ & - \\
\hline SKNMC junction probes & & & + \\
\hline Ciz1-ex8a0-ex12a1 & + & + & $=$ \\
\hline Ciz1-ex8af2-ex15 & + & + & + \\
\hline Ciz1-ex12-ex13 & + & + & $=$ \\
\hline Ciz1-ex15-ex16 & - & $=$ & \\
\hline
\end{tabular}

Three independent RNA isolates from proliferating SKNMC cell (Biological replicates, B1, B2 and B3) were analysed on individual arrays, with one isolate undergoing analysis in triplicate (technical replicate, $\mathrm{T} 1, \mathrm{~T} 2$ and $\mathrm{T} 3$ shown in Fig. $2 \mathrm{~A}$ and 3). Probes that are up or down regulated more that 1.5 fold and who's SEM indicate agreement between three technical replicates are shown. Results for these probes derived from the two further biological replicates (replicate 2 and replicate 3) are also shown. + indicates significantly up-regulated, - indicates significantly down-regulated and = indicates no significant change. Significance is taken to be a change that is $\geq 1.5$ fold compared to control RNA. For paediatric cancer cell line SKNMC, expression of two probes was consistently and significantly altered compared to control samples, these are Ciz1-ex8a0-ex12a1 and Ciz1-ex12-ex13. Raw data is given in Additional file 5, tables S4-S8.

been reported that alternative 5' UTR influences splicing and may alter protein function [20]. Furthermore, inappropriate expression of $5^{\prime}$ UTRs has been implicated in carcinogenesis in the case of BRCA1 [21] and Mdm2 [22]. The functional significance of CIZ1 alternative exon 1 expression requires further investigation, however their expression may play a role in specifying alternative splicing of the rest of the CIZ1 gene.

\section{Exclusion of exon 4}

Previously, we showed that an alternatively spliced human CIZ1 variant, with deletion of exon $4(\Delta \mathrm{E} 4)$, is mis-expressed as a consequence of intronic mutation in Ewings Tumour (ET) cell lines, including the two cell lines used here [9]. However, skipping of exon 4 was not reproducibly detected in either cell line. This is most likely due to constraint inherent in the sequence of

Table 3 Summary of $\mathrm{CIZ1}$ alternative splicing in TTC466 Ewing cell line

\begin{tabular}{|c|c|c|c|}
\hline TTC466 exon probes & $\begin{array}{l}\text { Average of } 3 \text { technical replicates } \\
\text { (Biol. 1) }\end{array}$ & $\begin{array}{l}\text { Biological } \\
\text { replicate } 2\end{array}$ & $\begin{array}{l}\text { Biological } \\
\text { replicate } 3\end{array}$ \\
\hline Ciz1-ex1 & + & nd & $=$ \\
\hline Ciz1-ex1b1 & + & $=$ & $=$ \\
\hline Ciz1-ex1c & + & + & + \\
\hline Ciz1-ex2 & + & $=$ & $=$ \\
\hline Ciz1-ex3 & + & + & + \\
\hline \multicolumn{4}{|c|}{ TTC466 junction probes } \\
\hline Ciz1-ex1ca1-ex2 & + & + & + \\
\hline Ciz1-ex1c-ex2a2 & + & + & + \\
\hline Ciz1-ex1c-ex4 & + & + & + \\
\hline Ciz1-ex1c-ex6 & + & + & + \\
\hline Ciz1-ex2-ex7-a & + & + & + \\
\hline Ciz1-ex2-ex7-b & + & $=$ & $=$ \\
\hline Ciz1-ex4-ex5 & + & + & + \\
\hline Ciz1-ex7-ex10 & + & + & + \\
\hline Ciz1-ex8a0-ex12a1 & - & nd & - \\
\hline Ciz1-ex9-ex10 & + & + & + \\
\hline Ciz1-ex13-ex14 & + & $=$ & $=$ \\
\hline Ciz1-ex15-ex16 & - & $=$ & $=$ \\
\hline Ciz1-ex16-ex17 & + & $=$ & $=$ \\
\hline
\end{tabular}

Three independent RNA isolates from proliferating TTC466 cells (Biological replicates, B1, B2 and B3) were analysed on individual arrays, with one isolate undergoing analysis in triplicate (technical replicate, T1, T2 and T3). Probes that are up or down regulated more that 1.5 fold and who's SEM indicate agreement between three technical replicates are shown. Results for these probes derived from the two further biological replicates (replicate 2 and replicate 3 ) are also shown. + indicates significantly up-regulated, - indicates significantly down-regulated and = indicate no significant change. For paediatric cancer cell line TTC466, expression of 2 exon probes was consistently and significantly altered compared to control samples, these are Ciz1-ex1c and Ciz1-ex3. For exon junction probes a total of 8 probes met all our selection criteria. These are Ciz1-ex1ca1-ex2, Ciz1-ex1c-ex2a2, Ciz1-ex1c-ex4, Ciz1-ex1c-ex6, Ciz1-ex2-ex7-a, Ciz1-ex4-ex5, Ciz1-ex7ex10 and Ciz1-ex9-ex10. Raw data is given in Additional file 6, tables S9-S13. 
CIZ1 junction exon 3-exon 5. Thus, this approach is not suitable for further investigation of alternative splicing at this site.

\section{A novel cancer-associated variant}

In addition to extensive AS of exon 1c, for TTC4666 cells a further 9 probes were significantly under or over represented in 3 technical replicates compared to the reference control, and four of these (2-7a, 4-5, 7-10, 9-10) were reproducibly detected in 2 further biological replicates. We were most interested in one probe $(8 \mathrm{a} 0$ 12a1) that is down-regulated in 3 of 3 technical replicates and 2 of 3 biological replicates in TTC466 cells, because the same probe is up-regulated in all replicate experiments carried out with SKNMC cells. This is the only probe that is significantly altered in both cell lines, albeit upregulated in one and down regulated in the other. This probe indicates expression of a CIZ1 splice variant with partial deletion of exon 8 and 12 and skipping of exon 9, 10 and 11, and is the top one of 4 ranked products for SKNMC cells (additional file 4 Table S2).

To further investigate alternative splicing between exon 8 and 12 in human CIZ1 (Fig. 5A), we used RTPCR from exon 8 (forward primer $8 \mathrm{~F}$ ) coupled with a reverse primer in exon 13 (13R). Results showed 3 transcripts in Ewings tumour cell lines and also in a third

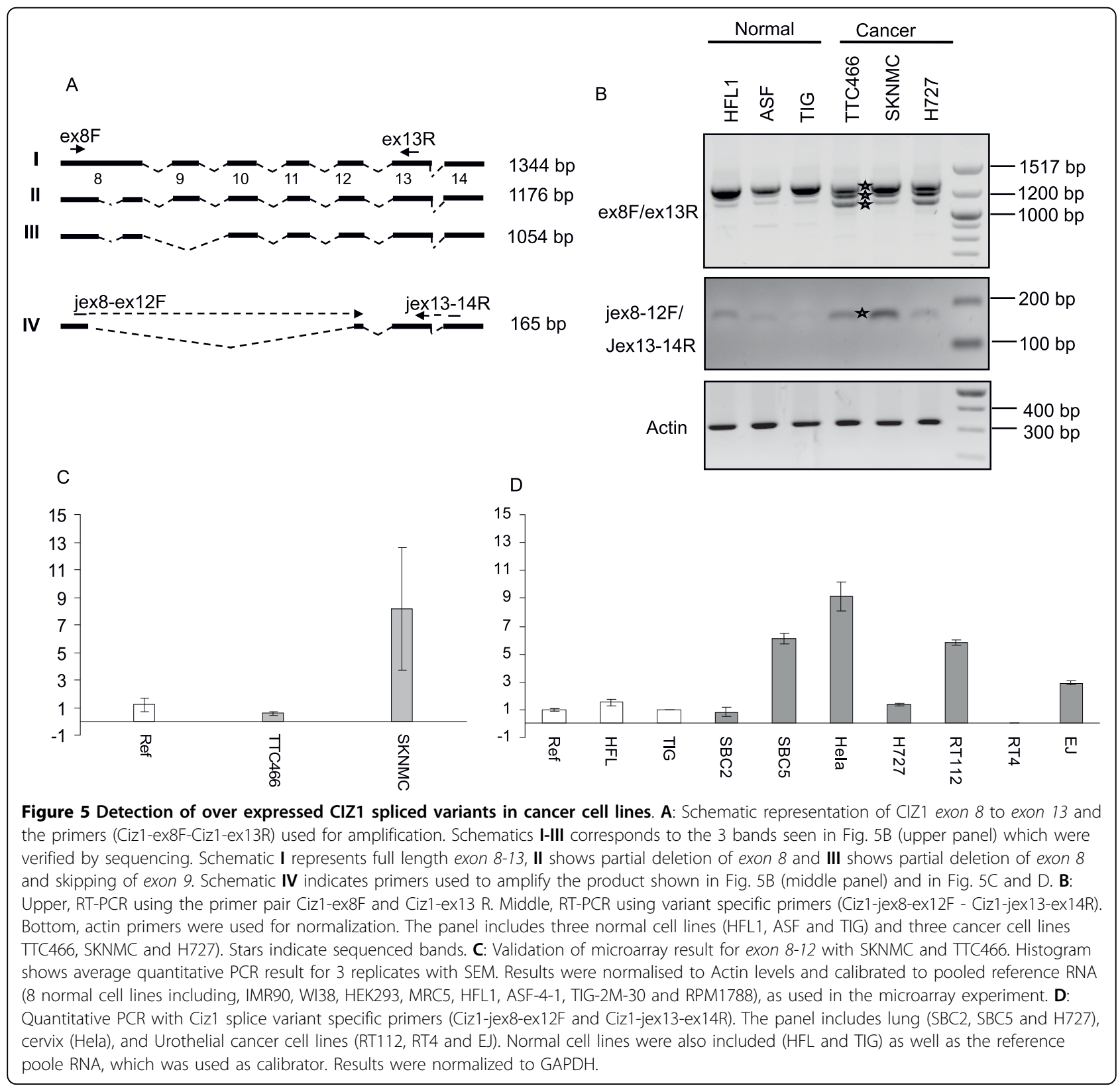


cancer cell line H727 (human lung carcinoid). Three products of the same mobility were detectable in 3 normal cell lines, but at considerably lower levels for the smaller of the three (Fig. 5B, upper). Thus, these products are over-expressed in cancer cell lines. Sequencing revealed the upper band (1344bp) to be the expected CIZ1 full-length sequence (Reference sequence NM_0121276.2, Fig. 5A), the middle band to lack sequences internal to exon 8 (168 bp) and the lower band to lack this region plus the whole of exon 9 (1054 bp). Taking NM_012127.2 as a reference sequence the exon 9 deletion is described as c.1338_1505del168 and exon 9 skipping as r.1702_1823del. Despite its prevalence in the cancer cell lines we studied, to our knowledge, this is the first time this variant has been reported. Neither of these events is represented by probes in our array. Notably, the variant detected by our array that splices exon 8 to exon 12 (c1038_2218del1181) was barely detectable by this analysis. Therefore in order to quantify its expression we designed specific primers Ciz1-jex8-ex12F-Ciz1-jex13-ex14R (Fig. 5A). Results showed increased expression in SKNMC cells compared to 3 normal cell lines (HFL, ASF and TIG) and comparable expression in TTC466 cells (Fig. 5B, middle). The $165 \mathrm{bp}$ product was sequenced, verified for SKNMC and TTC466 and found to be as expected. Similar results were obtained by quantitative real time PCR after normalisation to actin levels and calibration to the pooled reference RNA used in the arrays. As expected, results showed significant over expression for this variant (exon 8-exon 12) in SKNMC and slight down-regulation in TTC466 cell line (Fig. 5C), validating results obtained by array analysis. Quantitative detection of this apparently cancer-associated variant was extended to cover other cancer cell lines derived from lung (SBC2, SBC5 and H727), cervix (Hela) and urothelial cells (RT112, RT4 and EJ). Two individual normal cell lines HFL and TIG, as well as the pooled reference RNA were included as controls. Significant (greater than 2 fold) over- expression was detected in four of the 7 cancer cell lines (Fig. 5D). Furthermore, using Tissue Scan lung cancer array HLRT504 (Origene), we performed quantitative realtime PCR on cDNA obtained from 48 tissues. Results showed significantly elevated levels of this CIZ1 splice variant in 13 tumour samples compared to the matched paired normal controls (Fig. 6). Thus, the data indicate that exclusion of exon 9, 10, 11 and part of exon 8 and exon 12 is a recurrent event in cancer cells. This variant (c1038_2218del1181) lacks sequences that encode motifs that are important for the function of Ciz1 in DNA replication. Bioinformatic analysis revealed that the excluded sequence encodes as many as 6 putative CDK phosphorylation sites and 2 cyclin interaction sites (CY motifs). Notably sequences encoded by exon 9 engage in sequential interaction with cyclin $\mathrm{E}$ and $\mathrm{A}$, and support functional cooperation with cyclinA/CDK2 driving initiation of DNA replication in vitro [2]. This analysis was carried out with murine Ciz1 protein which is $64 \%$ homologous at the protein level to human Ciz1 in this region (exon 8-12). As the human variant described here

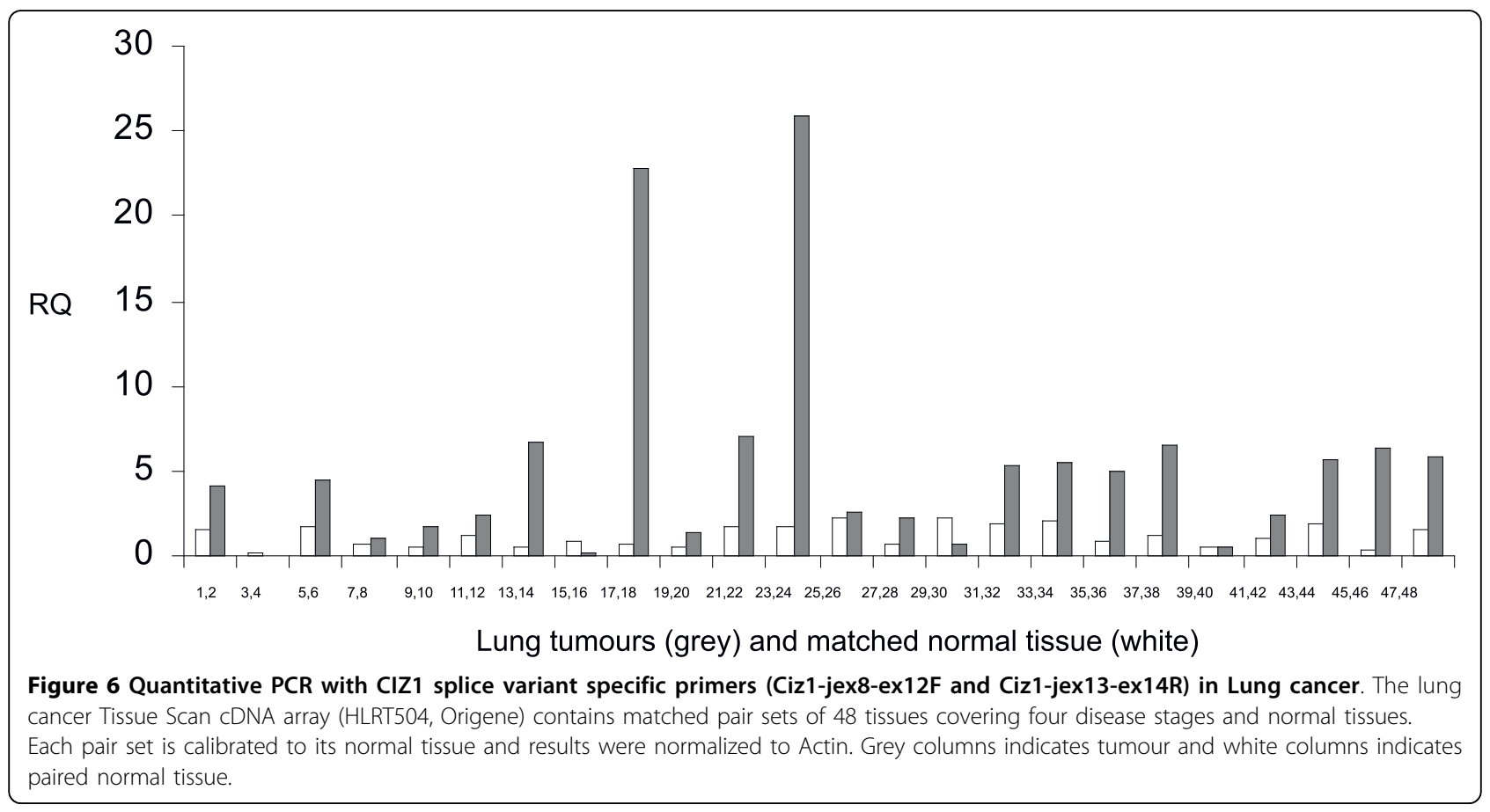


is lacking these sequences our extrapolation is that it will not be active in DNA replication, despite most likely retaining down-stream sequences that anchor Ciz1 to the nuclear matrix [6]. Although further functional analyses are needed to understand the impact that expression of this variant has on DNA replication, these data suggest it may interfere with the normal function of Ciz1.

\section{Conclusion}

Expression of variant 8a0-12a1 that is restricted to cancer cells may offer a useful marker for their detection. Furthermore, because siRNA depletion of Ciz1 has been shown to delay $\mathrm{S}$ phase entry and restrain cell proliferation [5], selective inhibition of CIZ1 variants have potential to offer selective restraint of those cell that express them. Thus variant $8 \mathrm{a} 0-12 \mathrm{a} 1$ has potential application as a selective therapeutic target in some lung cancer patients.

\section{Additional material}

\section{Additional file 1: Table S1. Probe sequences.}

Additional file 2: Figure S1. Schematic representation of predicted human Ciz1 alternatively spliced transcripts assembled by AceView from 865 mRNA and ESTs submitted to GenBank [17]. Blue lines represent exons, black represent introns, yellow represent $5^{\prime}$ or $3^{\prime}$ untranslated regions and * represent non canonical exon-intron boundaries. Alternative transcript assemblies were designated, $B, A, E, F, G, H, I, J, K, L$ $M, P, Q, R, S, V, W, Y, Z, 27,28,29,30,31$ and 32 by AceView.

Additional file 3: Figure S2. Observed and hypothetical exon-junctions generated by alternative splicing of Ciz1 alternative exon $1 \mathrm{~s}$ (ex 1a, ex $1 \mathrm{~b}$, ex $1 \mathrm{c}$ and ex 1d). Common exons are indicated by labelled boxes and less common alternative exons by solid black boxes. Observed and hypothetical junctions are indicated by black and grey broken lines respectively. Splicing events that are over represented in TTC466 are indicated by solid lines. Sequences at $3^{\prime}$ end of alternative exon $1 \mathrm{~s}$ are given in additional file 7 Table S14.

Additional file 4: Table S2. Ranked product, top 4 up-regulated probes in technical replicates of SKNMC. Table S3 Ranked product, top 4 upregulated probes in technical replicates of TTC466.

Additional file 5: Table S4. microarray result for SKNMC technical replicate 1 (T1). Table S5 microarray result for SKNMC technical replicate 2 (T2). Table S6 microarray result for SKNMC technical replicate 3 (T3). Table S7 microarray result for SKNMC biological replicate 2 (B2). Table S8 microarray result for SKNMC biological replicate 3 (B3).

Additional file 6: Table S9. microarray result for TTC466 technical replicate 1 (T1). Table $\mathbf{S 1 0}$ microarray result for TTC466 technical replicate 2 (T2). Table S11 microarray result for TTC466 technical replicate 3 (T3). Table $\mathbf{S 1 2}$ microarray result for TTC466 biological replicate 2 (B2). Table $\mathbf{S 1 3}$ microarray result for TTC466 biological replicate 3 (B3).

Additional file 7: Table S14. Sequences of alternative exon 1 ( $3^{\prime}$ end)

\section{Abbreviations}

PNET: primitive neuroectodermal tumor; Ciz1: CDKN1A interacting zinc finger protein 1; RT-PCR: reverse transcriptase polymerase chain reaction; AS: alternative splicing; EST: expressed sequence tag; BLAST: Basic Alignment Search Tool; BLAT: Blast-Like Alignment Tool; cDNA: DNA complementary to
RNA; NEB: New England Biolab; RB1: retinoblastoma 1; VPS72: vacuolar protein sorting 72 homolog (S. cerevisiae); CDC2: cell division cycle 2; CDC6: cell division cycle 6 homolog (S. cerevisiae); TNRC9: TOX high mobility group box family member 3; DDX17: DEAD (Asp-Glu-Ala-Asp) box polypeptide 17: UTR: Un-Translated Region; BRCA1: breast cancer 1; Mdm2: Murine double minute 2; SEM: Standard Error of the Mean; GAPDH: glyceraldehyde-3phosphate dehydrogenase.

\section{Acknowledgements}

This work was funded wholly by Yorkshire Cancer Research (registered charity 516898). All authors are employees of the University of York. We thank N Copeland and J Ainscough for critical comments on the manuscript.

\section{Author details}

${ }^{1}$ Department of Biology, University of York, UK. ${ }^{2}$ Parkwood Hospital, Blackpool, UK.

\section{Authors' contributions}

FAR participated in the design of the study, carried out molecular genetic studies, statistical analysis, data interpretation and drafted the manuscript. NA participated in the design of the study, statistical and data interpretation. DC conceived of the study, and participated in its design and coordination and helped to draft the manuscript. All authors read and approved the final manuscript.

\section{Competing interests}

DC is partly supported by and holds shares in University of York spin-out Cizzle Biotechnology. The CIZ1 splice variant array, and the delta 8-12 variant described in this paper were patent protected by the University of York on 30 July 2009.

Received: 28 August 2009 Accepted: 10 September 2010

Published: 10 September 2010

\section{References}

1. Warder DE, Keherly MJ: Ciz1, Cip1 interacting zinc finger protein 1 binds the consensus DNA sequence ARYSR(0-2)YYAC. Journal of Biomedical Science 2003, 10:406-417.

2. Copeland NA, Sercombe HE, Ainscough J, F-X, Coverley D: Ciz1 cooperates with cyclin $\mathrm{A} / \mathrm{CDK} 2$ to activate mammalian DNA replication in vitro. Journal of Cell Science.

3. Mitsui K, Matsumoto A, Ohtsuka S, Ohtsubo M, Yoshimura A: Cloning and characterization of a novel P21 Cip1/Waf1 - interacting zinc finger protein, Ciz1. Biochemical and Biophysical Research Communications 1999, 264:457-464.

4. Coverley D, Laman H, Laskey RA: Distinct roles for cyclins E and A during DNA replication complex assembly and activation. Nature Cell Biology 2002, 4:523-528.

5. Coverley D, Marr J, Ainscough JF-X: Ciz1 promotes mammalian DNA replication. Journal of Cell Science 2005, 118:101-112.

6. Ainscough JF, Rahman FA, Sercombe H, Sedo A, Gerlach B, Coverley D: Cterminal domains deliver the DNA replication factor $\mathrm{Ciz} 1$ to the nuclear matrix. J Cell Sci 2007, 120:115-124.

7. den Hollander P, Rayala SK, Coverley D, Kumar R: Ciz1, a novel DNAbinding coactivator of the estrogen receptor $\alpha$, confers hypersensitivity to estrogen action. Cancer Research 2006, 66:11021-11030.

8. Hakim O, John S, Ling JQ, Biddie SC, Hoffman AR, Hager GL: Glucocorticoid receptor activation of the Ciz1-Lcn2 locus by long range interactions. J Biol Chem 2009, 284:6048-6052.

9. Rahman FA, Ainscough JF-X, Copeland N, Coverley D: Cancer-associated missplicing of exon 4 influences the subnuclear distribution of the DNA replication factor Ciz1. Human Mutation 2007, 28:993-1004.

10. Dahmcke CM, Buchmann-Moller S, Jensen NA, Mitchelmore C: Altered splicing in exon 8 of the DNA replication factor $\mathrm{ClZ1}$ affects subnuclear distribution and is associated with Alzheimer's disease. Mol Cell Neurosci 2008, 38:589-594.

11. Kent WJ, Sugnet CW, Furey TS, Roskin KM, Pringle TH, Zahler AM, Haussler D: The human genome browser at UCSC. Genome Res 2002, 12:996-1006 
12. Srinivasan $K$, Shiue L, Hayes JD, Centers R, Fitzwater $S$, Loewen $R$, Edmondson LR, Bryant J, Smith M, Rommelfanger C, Welch V, Clark TA, Sugnet CW, Howe KJ, Mandel-Gutfreund Y, Ares M Jr: Detection and measurement of alternative splicing using splicing-sensitive microarrays. Methods 2005, 37:345-359.

13. Smith AFA, Hubley R, Green P: RepeatMasker Open-3.0 1996.

14. Altschul SF, Madden TL, Schaffer AA, Zhang J, Zhang Z, Miller W, Lipman DJ: Gapped BLAST and PSI-BLAST: a new generation of protein database search programs. Nucleic Acids Res 1997, 25:3389-3402.

15. Le K, Mitsouras K, Roy M, Wang Q, Xu Q, Nelson SF, Lee C: Detecting tissue-specific regulation of alternative splicing as a qualitative change in microarray data. Nucleic Acids Res 2004, 32:e180.

16. Richmond CS, Glasner JD, Mau R, Jin H, Blattner FR: Genome-wide expression profiling in Escherichia coli K-12. Nucleic Acids Res 1999, 27:3821-3835.

17. Thierry-Mieg D, Thierry-Mieg J: AceView: a comprehensive cDNAsupported gene and transcripts annotation. Genome Biol 2006, 7(Suppl 1 (S12)):11-14.

18. Breitling R, Armengaud P, Amtmann A, Herzyk P: Rank products: a simple, yet powerful, new method to detect differentially regulated genes in replicated microarray experiments. FEBS Lett 2004, 573:83-92.

19. Rual JF, Venkatesan K, Hao T, Hirozane-Kishikawa T, Dricot A, Li N, Berriz GF, Gibbons FD, Dreze M, Ayivi-Guedehoussou N, Klitgord N, Simon C, Boxem M, Milstein S, Rosenberg J, Goldberg DS, Zhang LV, Wong SL, Franklin G, Li S, Albala JS, Lim J, Fraughton C, Llamosas E, Cevik S, Bex C, Lamesch P, Sikorski RS, Vandenhaute J, Zoghbi HY, Smolyar A, Bosak S, Sequerra R, Doucette-Stamm L, Cusick ME, Hill DE, Roth FP, Vidal M: Towards a proteome-scale map of the human protein-protein interaction network. Nature 2005, 437:1173-1178.

20. Smith L: Post-transcriptional regulation of gene expression by alternative 5 '-untranslated regions in carcinogenesis. Biochem Soc Trans 2008, 36:708-711.

21. Sobczak K, Krzyzosiak WJ: Structural determinants of BRCA1 translational regulation. J Biol Chem 2002, 277:17349-17358.

22. Okumura N, Saji S, Eguchi H, Nakashima S, Saji S, Hayashi S: Distinct promoter usage of mdm2 gene in human breast cancer. Oncol Rep 2002, 9:557-563.

23. Zhang J, Hu S, Schofield DE, Sorensen PH, Triche TJ: Selective usage of DType cyclins by Ewing's tumors and rhabdomyosarcomas. Cancer Res 2004, 64:6026-6034.

24. Dunn T, Praissman L, Hagag N, Viola MV: ERG gene is translocated in an Ewing's sarcoma cell line. Cancer Genet Cytogenet 1994, 76:19-22.

25. Takahashi T, Nau MM, Chiba I, Birrer MJ, Rosenberg RK, Vinocour M Levitt M, Pass H, Gazdar AF, Minna JD: p53: a frequent target for genetic abnormalities in lung cancer. Science 1989, 246:491-494.

26. Nichols WW, Murphy DG, Cristofalo VJ, Toji LH, Greene AE, Dwight SA: Characterization of a new human diploid cell strain, IMR-90. Science 1977 196:60-63.

27. Hayflick L, Moorhead PS: The serial cultivation of human diploid cell strains. Exp Cell Res 1961, 25:585-621.

28. Shaw G, Morse S, Ararat M, Graham FL: Preferential transformation of human neuronal cells by human adenoviruses and the origin of HEK 293 cells. Faseb J 2002, 16:869-871.

29. Jacobs JP, Jones CM, Baille JP: Characteristics of a human diploid cell designated MRC-5. Nature 1970, 227:168-170.

30. Breul SD, Bradley KH, Hance AJ, Schafer MP, Berg RA, Crystal RG: Control of collagen production by human diploid lung fibroblasts. J Biol Chem 1980, 255:5250-5260.

31. Kaji K, Kanda N: Cellular aging and growth characteristics of two human skin fibroblast strains, derived from a same individual at different ages of a 11 years interval. BiomedGerentol 1992, 16:132.

32. Rubinstein HM, Lubrano T, Mathews HL, Lange CF, Silberman S, Adams EM, Minowada J: A lymphocyte cell line that makes serum cholinesterase instead of acetylcholinesterase. Biochem Genet 1984, 22:1171-1175.

33. Aizawa S, Mitsui $Y$, Kurimoto F, Matsuoka K: Cell-surface changes accompanying aging in human diploid fibroblasts: effects of tissue, donor age and genotype. Mech Ageing Dev 1980, 13:297-307.

\section{Pre-publication history}

The pre-publication history for this paper can be accessed here:

http://www.biomedcentral.com/1471-2407/10/482/prepub doi:10.1186/1471-2407-10-482

Cite this article as: Rahman et al.: Differential detection of alternatively spliced variants of Ciz1 in normal and cancer cells using a custom exon-junction microarray. BMC Cancer 2010 10:482.

\section{Submit your next manuscript to BioMed Central and take full advantage of:}

- Convenient online submission

- Thorough peer review

- No space constraints or color figure charges

- Immediate publication on acceptance

- Inclusion in PubMed, CAS, Scopus and Google Scholar

- Research which is freely available for redistribution 